\title{
Tehran dentists' self-reported knowledge and attitudes towards HIV/AIDS and observed willingness to treat simulated HIV-positive patients
}

B. Khosravanifard, ${ }^{7}$ V. Rakhshan, ${ }^{2}$ M. Ghasemi, ${ }^{3}$ A. Pakdel, ${ }^{4}$ S. Baradaran-Eghbal, ${ }^{5}$ R. Sheikholeslami, ${ }^{5}$ T. DadolahiSarab ${ }^{5}$ and H. Rakhshan ${ }^{5}$

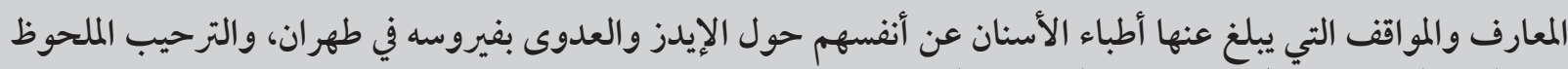

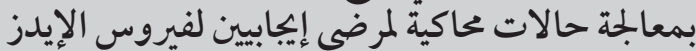

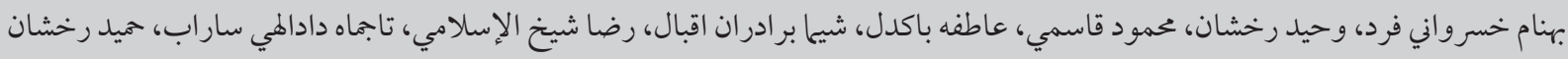

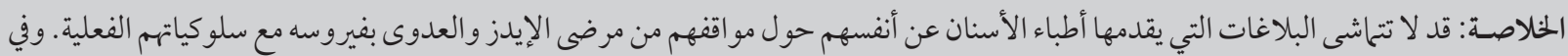

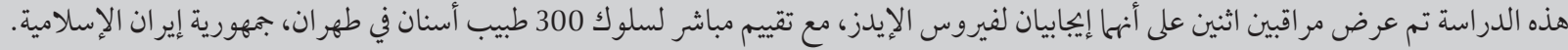

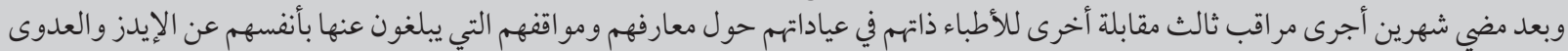

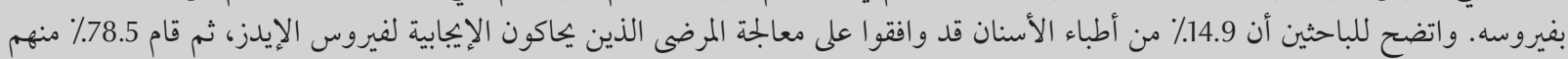

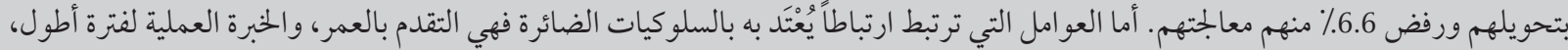

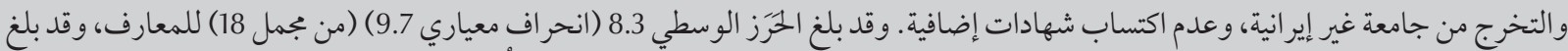

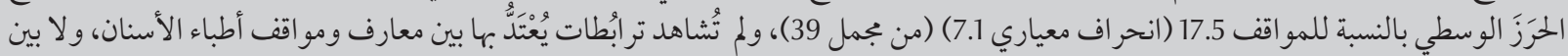

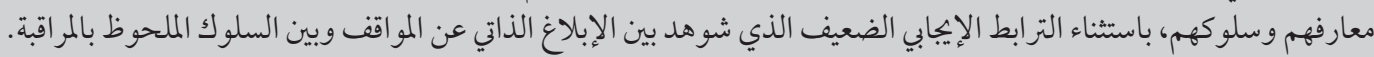

ABSTRACT Dentists' self-reported attitudes towards patients with HIV/AIDS might not reflect their actual behaviour. In this study 2 observers posed as HIV-positive patients and directly evaluated the behaviour of 300 dentists in Tehran, Islamic Republic of Iran. Two months later another observer interviewed the same dentists at their offices regarding AIDS-related knowledge and self-reported attitudes. Only $14.9 \%$ of dentists agreed to treat the simulated HIV-positive patients, 78.5\% referred and 6.6\% rejected them. Older age, longer work experience, graduation from a non-Iranian university and not having additional degrees were significantly related to adverse behaviours. Mean scores were 8.3 (SD 9.7) (out of 18) for knowledge and 17.5 (SD 7.1) (out of 39) for attitude. There were no significant correlations between dentists' knowledge and attitude or between knowledge and behaviour, but there was a weak positive correlation between self-reported attitude and observed behaviour

Connaissances et attitudes autodéclarées des dentistes à Téhéran par rapport au VIH/sida et disposition observée à soigner les patients se présentant comme positifs pour le VIH

RÉSUMÉ II est possible que l'attitude autodéclarée des dentistes vis-à-vis des patients atteints du VIH/sida ne corresponde pas à leur comportement en situation réelle. Dans le cadre de cette étude, deux observateurs se sont fait passer pour des patients séropositifs pour le VIH et ont directement évalué le comportement de 300 dentistes de Téhéran (République islamique d'Iran). Deux mois plus tard, un autre observateur a interrogé les mêmes dentistes dans leur cabinet au sujet de leurs connaissances et de leur attitude autodéclarées à propos du sida. Seuls $14,9 \%$ des dentistes ont consenti à traiter les patients ayant déclaré une séropositivité fictive, 78,5\% les ont orientés vers d'autres confrères et 6,6 \% ont refusé de les soigner. Un âge plus avancé, une expérience professionnelle plus longue, des études suivies dans une université non iranienne et le fait de ne pas avoir de diplômes supplémentaires étaient des facteurs significativement associés à des comportements de refus. Les scores moyens étaient de 8,3 (E.T. 9,7 ) (sur 18) pour les connaissances et de 17,5 (E.T. 7,1) (sur 39) pour l'attitude. Aucune corrélation significative n'a été observée entre les connaissances et les attitudes des dentistes ni entre les connaissances et le comportement, mais une faible corrélation positive a été retrouvée entre l'attitude autodéclarée et le comportement observé.

${ }^{7}$ Department of Orthodontics; ${ }^{2}$ Department of Dental Anatomy and Morphology; ${ }^{3}$ Department of Periodontics; ${ }^{4}$ Department of Paediatric Dentistry; Dental Branch, Islamic Azad University, Tehran, Islamic Republic of Iran. ${ }^{5}$ Private Practice, Tehran, Islamic Republic of Iran (Correspondence to Vahid Rakhshan: vahid.rakhshan@gmail.com).

Received: 24/03/11; accepted: 16/05/11 


\section{Introduction}

Dental professionals are at a high risk for cross-infection with HIV [1] and may therefore avoid treating HIV-positive dental patients [2]. This has serious public health consequences. For instance, in $60 \%$ to $70 \%$ of HIV-positive individuals oral symptoms of HIV infection are the first signs of the syndrome appearing and these can be used as appropriate diagnostic criteria in the detection of AIDS [3]. Additionally, healthy asymptomatic HIV-positive dental patients may conceal their condition in order to receive appropriate treatment, which may have serious consequences such as increased risk of cross-contamination [4].

The attitudes of medical health professionals towards treating patients living with HIV/AIDS seem to have changed over time and there appear to be a wide range of attitudes across different cultures [5-20]. There is also evidence that legal measures alone cannot change health professionals' attitudes towards HIV patients. Improved awareness of dentistry personnel with respect to HIV carriers and the likelihood of infection might play a positive role in improving dentists' attitude and confidence towards treating patients with HIV [5,15]. However, previous studies worldwide of dentists' attitudes towards treating HIV/AIDS-positive patients were based only on dentists' or patients' declarations, which might not necessarily be in line with clinicians' actual behaviours, and no studies have directly evaluated actual behaviours/reactions (practices) of dentists during visits by HIV/AIDS-positive patients. Furthermore, no similar studies have been performed on a sample of Tehran dentists. We aimed to evaluate Tehran dentists' practices directly using simulated patients [21] and then assess their AIDS-related knowledge and their declared attitudes towards treating AIDS patients in face-to-face interviews. This was the first study of its kind and it was hoped that the information would be of value for alerting the authorities to allocate educational or infection control resources more effectively and to avoid discrimination against HIV patients.

\section{Methods}

This was a prospective, cross-sectional study carried out during 2008-09.

\section{Sample}

To obtain a test power of 0.85 , and based on a pilot study, an estimated 300 dentists were required to participate. A list of all dentists in Tehran was obtained from the Iranian Medical Council. Using simple random sampling from the alphabetic list of about 6100 Tehran dentists, and to allow for dropouts, a total of 411 dentists were randomly selected and visited. The final analysis was based on the 300 dentists (230 general practitioners and 70 specialists) who participated in both phases of the study.

\section{Data collection}

\section{Observed behaviour with simulated patients}

Two female dental students aged 22 and 23 years old were recruited as observers/simulated patients. An appointment for an oral examination session was made at each selected dentist by visiting or calling the dentist's office (each observer evaluated half the selected number of dentists). In the first session, just before the oral examination and/or when the patient's profile was being recorded, the observer claimed to the dentist or the assistant, both orally and in written form, to be bloodborne HIV-positive. The observer also ensured that the dentist was fully aware of the condition by telling him/her directly as well before any examinations. None of the clinicians suspected the observers were healthy researchers. The dentist's reaction was documented and the behaviour was classified as: accepting (accepted the patient and scheduled the next session) or nonaccepting (refused to admit the patient but referred to a specialist centre or rejected the patient without referring to other colleagues).

Thirty dentists were randomly selected to be assessed again, in terms of their reactions toward AIDS patients, by another observer about 1 month later. The reliability of dentists' behaviours was determined as $89 \%$ (Cohen $\mathrm{k}$ $=0.89, P=0.001$ ).

\section{Interviews using knowledge and atti- tude questionnaires}

After 2 months, a third observer called and visited the same dentists and asked to interview them face-to-face about their knowledge and attitudes using a structured questionnaire. The questionnaire was specially designed for this study by a panel of experts. The following items were documented: demographic data (age, sex); office location, based on income status categorized by Tehran municipality (Tehran south, west and east districts versus central and north district); office type (private office or general clinic with different departments); work experience; degrees obtained (Masters degree, degrees other than in the field of dentistry); country of graduation (Iranian versus foreign university); work experience; university staff member; continuing education courses (number of courses attended and time elapsed since last course).

The knowledge section of the questionnaire included 18 questions regarding diagnostic criteria and management of patients with HIV/AIDS (scored 1 or 0 ) [22-24]. The questions included the signs and symptoms of AIDS in dental patients, diagnosis of HIV and the latent period, methods of transmission, procedures for sterilization and avoidance of transmission, etc. The scores were stratified into 3 groups by a panel consisting of 8 specialists in 
dentistry and a statistician: good knowledge (score 15-18); average knowledge (score 10-15); poor knowledge (score $<10)$.

The attitudes section of the questionnaire included 13 questions with 4 Likert-scale answers which represented the extent of dentists' willingness to participate in treating AIDS patients (scored from 3 to 0 , i.e. certainly disagree, disagree, agree, certainly agree). The questions covered society's respect for and beliefs about AIDS patients, their own and other dentists' willingness and duty to treat AID patients, concerns about treating such patients, costs of treatment, etc. Attitudes were categorized into 3 groups: negative attitude (score $<20)$; average attitude (score 20-30); positive attitude (score 30-39).

\section{Ethical considerations}

The study was given ethical approval by the Research Committee of the Islamic Azad University, as well as the Medical Council of Iran. No personal identification or occupational data (i.e. names, phone numbers, exact addresses and license numbers) were collected. Each questionnaire included a consent form, in which the first phase (direct observation) was described to the dentists and they were assured that their personal information had not been collected.

\section{Statistical analysis}

Descriptive statistics were calculated. In the behaviour section, dentists were classified into accepting or nonaccepting groups. In the knowledge and attitude sections, the good and average groups were merged. The frequency distribution of each potentially correlating factor was calculated across the sample, then in order to dichotomize quantitative variables (e.g. dentist's age) to binominal ones, the distribution of each quantitative factor was divided into groups of greater and smaller than the rounded mean value of that factor.
Odds ratios (OR) and 95\% confidence intervals (CI) were calculated. Correlations between the variables and behaviour, knowledge and attitude were assessed using the chi-squared test. Spearman correlations (rho) were done using ranked scores for knowledge ( good $=2$, average poor $=1$ ) and for attitudes (positive $/$ average $=2$, negative $=1$ ). The level of significance was set at 0.05 .

\section{Results}

\section{Background characteristics}

Of the 411 dentists visited a total of 111 were unavailable or refused to participate in the second phase leaving 300 dentists for the analysis.

The mean age of the dentists was 46.5 (SD 19.1) years (range 27-64 years) and $71.0 \%$ of them were males; $86.3 \%$ worked in private offices, $68.3 \%$ worked in the centre or north of the city. Their mean work experience was 14.7 (SD 21.8) years (range 1-31); $7.0 \%$ had university teaching experience, $80.0 \%$ had graduated from Iranian universities, and $4.3 \%$ had other degrees as well as dentistry qualifications. The average years since the last continuing education course was 1.9 (SD 1.5) years (range 0-3).

\section{Observed behaviour}

Of the 300 dentists visited, 44 (14.7\%) accepted the simulated HIV-positive patientfor treatment, while 256 (85.3\%, 95\% CI: 80.9\%-88.9\%) refused. Of the non-accepting dentists, 236 (78.6\%) referred the patient elsewhere for treatment, while 20 (6.7\%) refused even to refer. Age, work experience, the country of graduation (Islamic Republic of Iran versus abroad) and having other degrees were significantly related to the dentists' practice (Table 1). Older dentists were significantly more unwilling to treat AIDS patients $(\mathrm{OR}=3.12$, 95\% CI: $1.08-10.0)$, and those who had studied at foreign universities were also less willing, which was borderline significant $(\mathrm{OR}=2.8,95 \% \mathrm{CI}$ : 0.96-8.16). In contrast, dentists with < 10 years work experience $(\mathrm{OR}=4.96$, 95\% CI: 2.30-10.7) and dentists with additional degrees other than dentistry ( $\mathrm{OR}=3.97,95 \% \mathrm{CI}: 1.24-12.8)$ were significantly more likely to agree to treat AIDS patients. Participants with experience of teaching at universities were also more willing, but not significantly so, to treat the simulated patients (OR $=2.54$, 95\% CI: 0.93-6.94) (Table 1).

\section{Self-reported knowledge}

In the knowledge section, 160 (53.3\%) of the 300 dental professionals who agreed to be interviewed scored poor (95\% CI: 47.7\%-58.9\%), 72 (24\%) average and $68(22.7 \%)$ good. The mean knowledge score was 8.3 (SD 9.7) (range 0-17), out of a maximum score of 18 . In the attitude section, 125 (41.7\%) of participants scored negative (95\% CI: 36.2\%-47.3\%), 174 (58\%) average and only $18(0.6 \%)$ positive.

Dentists' knowledge was significantly associated with the time since the last continuing education course (Table 1). Dentists not admitted to continuing education courses during the last 2 years were slightly but not significantly more likely to score better in knowledge ( $\mathrm{OR}=1.68,95 \% \mathrm{CI}=0.93-3.02)$. Faculty members were 2.4 times more likely to show better knowledge statuses but this was not statistically significant $(\mathrm{OR}=2.43,95 \%$ CI: $0.95-6.20)(P=$ 0.06) (Table 1).

\section{Self-reported attitudes}

The mean attitude score was 17.5 (SD 7.1) (range 5-33) out of 33. Dentists with good attitudes were significantly more likely to have graduated from an Iranian university than abroad ( $\mathrm{OR}=$ 3.33, 95\% CI: 1.84-6.01) (Table 1). Faculty members were more likely to show good attitudes ( $\mathrm{OR}=2.41,95 \%$ CI: 0.86-6.78), although this was not statistically significant. Those with work 


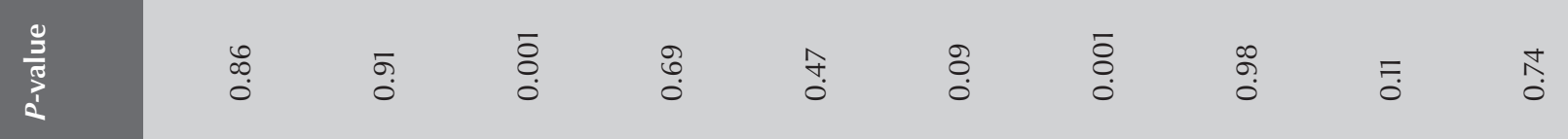

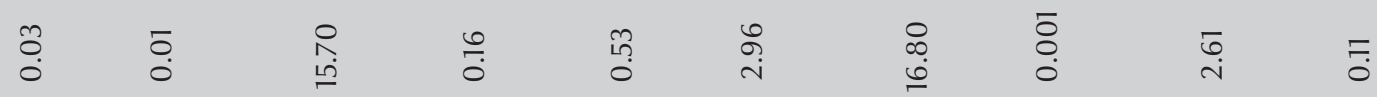

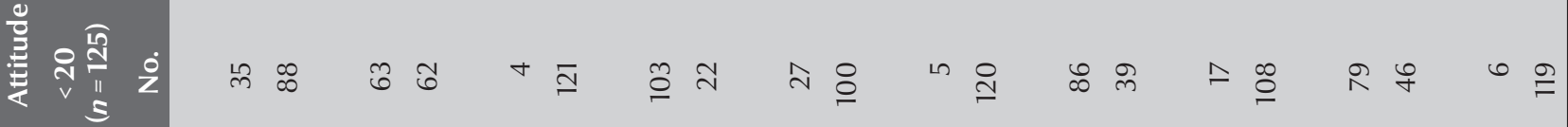

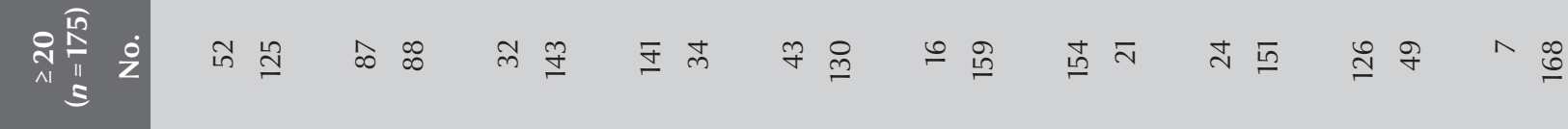
$\frac{\sqrt{2}}{\frac{0}{2}}$

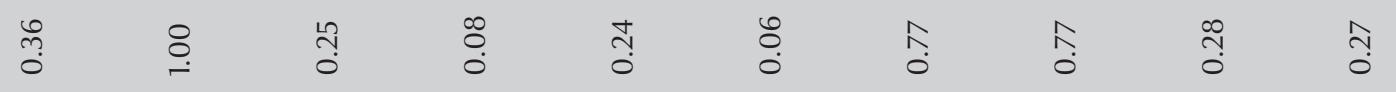
产

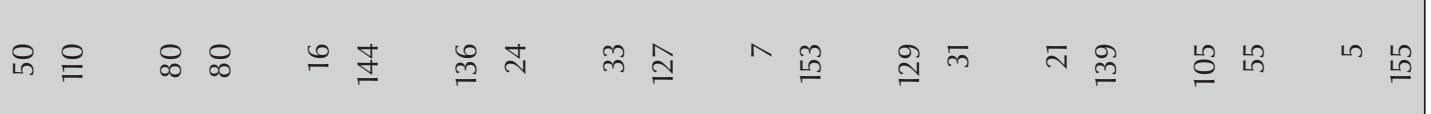

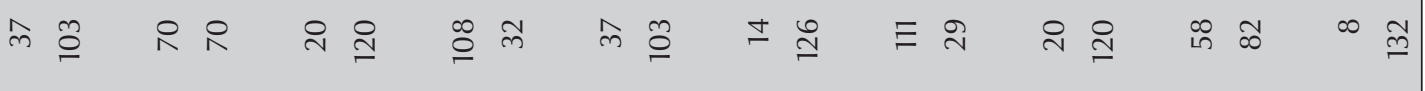

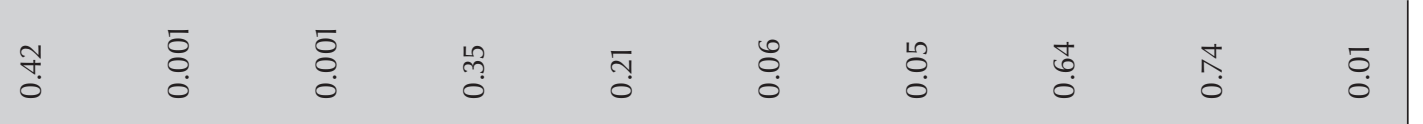

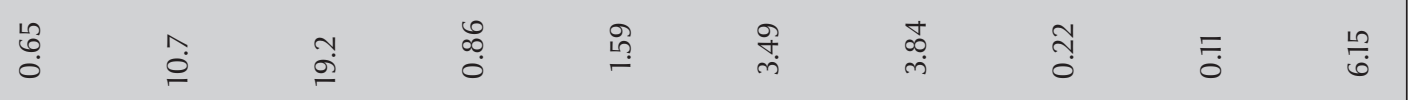

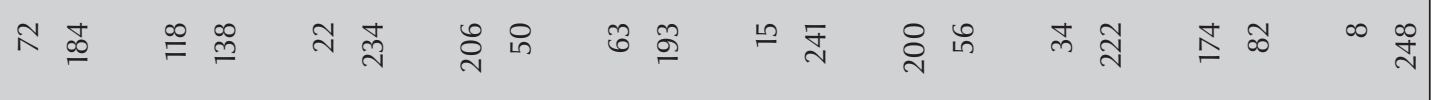
명

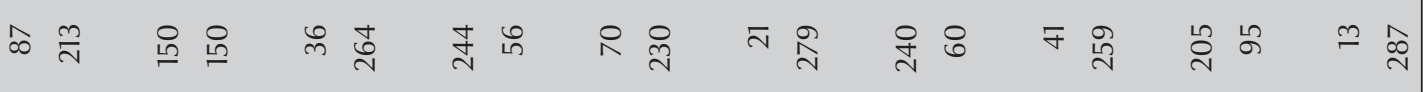

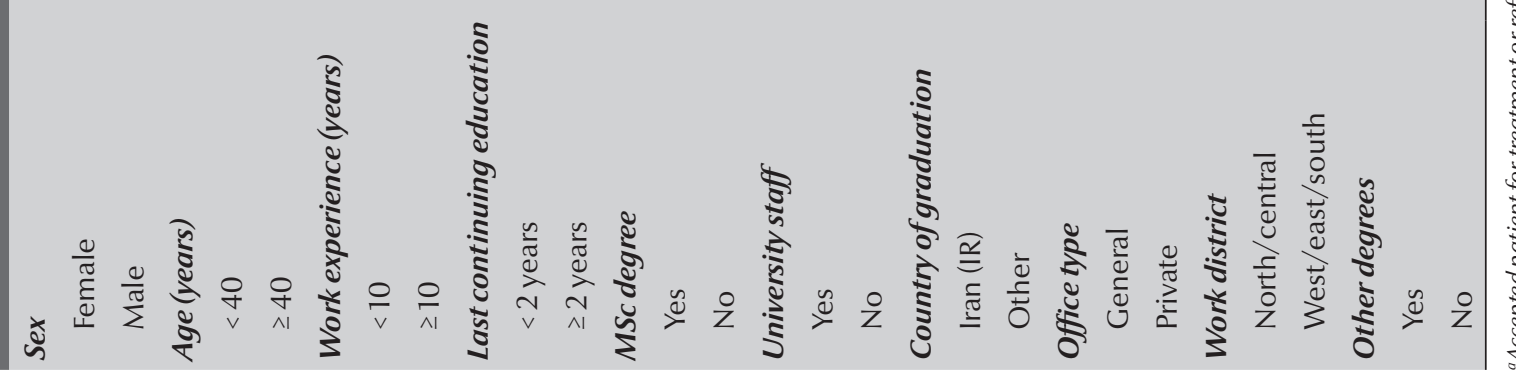


experience $<10$ years were 6.8 times more likely to admit AIDS patients (OR $=6.77,95 \%$ CI: $2.33-19.7)$. Dentists working in north and central Tehran districts had a tendency towards better attitudes $(\mathrm{OR}=1.50,95 \% \mathrm{CI}=$ 0.92-2.45).

\section{Correlations between knowledge, attitude and practice}

The Spearman correlation coefficient did not indicate any significant correlations between dentists' knowledge and attitude (rho $=0.102, P=0.077$ ) or between knowledge and behaviour (rho $=0.008, P=0.367)$. However, a significant (but weak) positive correlation was found between attitude and observed behaviour $(\mathrm{rho}=0.379, P=0.02$ ).

\section{Discussion}

In the present study, a great number of dentists in Tehran failed to score appropriately in knowledge, attitude and practices toward patients with HIV. Dentists' age, work experience, their country of graduation, and having degrees other than dental degrees significantly correlated with their observed practice in accepting or refusing to treat simulated patients.

Our results of direct observations showing that $14.9 \%$ of dentists agreed to treat the simulate patients were very different from the results of Hazelkorn, who reported a $99 \%$ rate of acceptance to treat homosexual dental patients in 1989 in the United States [21]. However, that study only assessed dentists' behaviours when facing a dental patient with a higher risk of having AIDS, not an HIV-positive patient. Besides, the extent of the AIDS epidemic and the negative attitudes towards it may have been different in that time and locality.

Our results concerning dentists' knowledge about HIV/AIDS were generally poorer than in other published studies $[5,8-10]$. The difference in subjects' knowledge in various investigations worldwide might be attributed to the effect of education via the media (e.g. television, radio), which vary across countries, and the younger ages evaluated in those studies. The literature indicates that younger dentists' attitudes and knowledge might be better $[6,9,13]$. However, comparison between the results from other settings is difficult as the studies have not used uniform methods/questionnaires to evaluate participants' attitudes or knowledge. The attitudes of medical health professionals (dentists, physicians and other health care staff) towards treatment of AIDS patients seem to have changed over time and there might be cultural differences in attitudes too. The findings in the literature vary widely, ranging from positive attitudes or appropriate knowledge - in Japan, 1997 [5], Mexico, 1998 [6], UK, 2005 [7], Nigeria, 2009 [8], Islamic Republic of Iran, 2009 [9] and Turkey, 2010 [10] - to relatively negative attitudes or poor knowledge observed in populations selected from USA, 1989 to 2006 [11-15], Japan, 2006 [16], Islamic Republic of Iran, 2006 [17], Nigeria, 2007 [18], UK, 2008 [19], and the Netherlands, 2008 [20].

Even allowing for difference in the questionnaires and methods, the rate of positive attitudes towards delivering oral health services to AIDS patients in the present study was considerably lower than in many other studies $[5-7,9-11,16,17]$, similar to certain studies $[7,16]$ and greater than the remainder $[6,15,17]$. This might be due to a combination of factors including sample differences such as mean ages, population sizes of the cities in which the studies were conducted (e.g. Tehran versus Shiraz [17] versus Mexico city [6]), differences in the level of education (e.g. dentists $[6,7,17]$, dental students $[10,14]$, dental residents [12], medical students $[10,14]$ or dental faculty [13]), cultural differences among different countries (e.g. attitudes towards having sexual relationships with various partners in different cultures), frequency of AIDS-related education via the media [5] (e.g. in the Islamic Republic of Iran versus in Japan with a 94\% rate of AIDS-oriented education via television) and the year of the study (e.g. in the early years of AIDS epidemic [21] versus 2008-09 in the present study). There were also methodological differences between these studies, which used mass surveys such as interviewing with mail or during conferences [7], interviews at dental offices (the present study) and direct observations [21].

A negative correlation was found between dentist's age and his/her observed reaction to an HIV-positive patient. However, data derived from the questionnaires failed to reveal any significant correlation between age and dentists' knowledge and attitudes. Therefore, in spite of their self-reported attitudes and knowledge, which were not significantly different from younger dentists, older dentists might be less financially interested in difficult cases, have higher workloads or be more disinclined to perform standard and appropriate infection control protocols. Other questionnaire-based studies agree with this finding, showing non-significant effects of dentists' age and work experience $[8,13,14,17]$ and a significant effect of dentists' sex on self-reported attitude $[8,12,13,25]$; however, there are some controversies over the effect of age $[7,10]$ and sex $[13,14]$. The same reasoning might account for the lower tendency of more experienced dentists (who might have busier offices and/or be older) to treat HIV-positive patients. Possession of degrees other than dentistry was associated with more favourable behaviour among clinicians. Further studies are needed to understand more about the reasons for this. In the present study, unlike the age factor, the country of graduation (Islamic Republic of Iran 
or abroad) significantly affected clinicians' practices, both self-reported and observed. However, despite their poorer behaviour and attitudes, dentists who studied abroad had appropriate knowledge comparable to the homeland-graduated dentists. Hence, their poorer attitude and behaviour scores might be rooted in differences in the prevalence of AIDS in certain countries (e.g. Philippines or Russia) which might foster greater concern about treating HIV patients among dentists graduated from those countries.

In contrast to research conducted in Japan [5], we found no significant correlation between dentists' knowledge with their attitudes and behaviours. Knowledge may raise practitioners' confidence to ignore negative messages regarding patients with HIV/AIDS; however, knowing how to manage and treat such patients is not the only factor a clinician may consider when deciding whether to treat a patient. Unlike many other countries, the main carriers of HIV in the Islamic Republic of Iran are intravenous drug users. This may give rise to concerns that the HIV-positive patientisfinancially and ethically impoverished, leading to further problems for the dentist, which might also influence his/her decision to treat. Furthermore, elementary standard precautions might be absent in private dental practices [17], which is a risk when dealing with AIDS patients. The infection control protocols needed for AIDS patients raise the cost of treatment, which might create a disincentive to treat them for some clinicians. Dentists should be taught that universal precautions should be used with all patients, since dentists and patients themselves will not always be aware of who is HIV-positive. Therefore, although knowledge is necessary to improve attitudes [15], it may not be sufficient. The weak association found between the results of direct observation and dentists' claimed attitudes in this study highlights the low reliability of self-reporting and the need for direct assessment methods in such surveys.

The face-to-face interview and direct survey approaches used in this study seem to be capable of obtaining more reliable results compared with designs based on mass data collection methods. However, this design is limited by factors such as being much slower to complete as well as the presence of observers' subjective bias, although the latter was reduced by determining clear-cut behaviour categories. The observers might experience difficulties in pretending to be HIV-positive or face inappropriate behaviours of some dental personnel after revealing their HIV-positive status. Indeed several dentists expressed their anger over the observers and some used impolite language. Some dental professionals suspected the third observer was a government investigator or tax collector, which was a factor in their refusal to participate and the subsequent loss of some data collected in the first phase. Another criticism of the study design is that during the first phase the dentists were deceived, which might give rise to ethical concerns. However, their personal data were not collected, and signed consent forms were taken from them in the second phase, after thoroughly describing the study. It might have been better to recruit trained actors as simulated patients [21] or actual HIV-positive patients. However, the students successfully convinced all the dentists they were AIDS patients. Another limitation of all of the studies in the field is the lack of standard questionnaires, which reduces the comparability of the results. In this study, simple random sampling was used due to its convenience; a stratified random sampling method would be more representative of the population.

\section{Conclusions}

Declared attitudes of dentists towards AIDS patients seemed to differ from their actual behaviours. A high proportion of Tehran dentists were unwilling to provide oral health care to AIDS patients, suggesting that there should be better legal provisions for AIDS patients facing discrimination [15]. Educational programmes are needed to improve attitudes of dentistry personnel-especially those who are older, more experienced and foreign-graduatedtowards patients with HIV/AIDS and improve knowledge about the risks of transmission $[5,10]$.

\section{References}

1. Greenberg M, Glick M. Burket's oral medicine: diagnosis and treatment. New York, BC Decker, 2003.

2. Seacat JD, Litt MD, Daniels AS. Dental students treating patients living with HIV/AIDS: the influence of attitudes and HIV knowledge. Journal of Dental Education, 2009, 73:437-444.

3. Lewis DA et al. Description and evaluation of an education and communication skills training course in HIV and AIDS for dental consultants. European Journal of Dental Education, 2000, 4:65-70.

4. Bharat S, Aggleton P, Tyrer P. India: HIV and AIDS-related discrimination, stigmatization and denial. UNAIDS best practice col- lection key material. Geneva, Joint United Nations Programme on HIV/AIDS, 2001 (UNAIDS/0146 E).

5. Kitaura $\mathrm{H}$ et al. Knowledge and attitudes of Japanese dental health care workers towards HIV-related disease. Journal of Dentistry, 1997, 25:279-283.

6. Irigoyen M, Zepeda M, López-Cámara V. Factors associated with Mexico City dentists' willingness to treat AIDS/HIV-positive patients. Oral Surgery, Oral Medicine, Oral Pathology, Oral Radiology, and Endodontics, 1998, 86:169-174.

7. Crossley ML. An investigation of dentists' knowledge, attitudes and practices towards HIV+ and patients with other blood- 
borne viruses in South Cheshire, UK. British Dental Journal, 2004, 196:749-754.

8. Uti OG et al. Infection control knowledge and practices related to HIV among Nigerian dentists. Journal of Infection in Developing Countries, 2009, 3:604-610.

9. Sadeghi M, Hakimi H. Iranian dental students' knowledge of and attitudes towards HIV/AIDS patients. Journal of Dental Education, 2009, 73:740-745.

10. Turhan $\mathrm{O}$ et al. Knowledge, attitudes and behaviour of students from a medicine faculty, dentistry faculty, and medical technology Vocational Training School toward HIV/AIDS. International Journal of Occupational Medicine and Environmental Health, 2010, 23:153-160.

11. Wallack JJ. AIDS anxiety among health care professionals. Hospital and Community Psychiatry, 1989, 40:507-510.

12. Cohen LA et al. Attitudes of advanced dental education students toward individuals with AIDS. Journal of Dental Education, 2005, 69:896-900.

13. Cohen LA, Romberg E, Grace E. Revisiting the attitudes of dental faculty toward individuals with AIDS. Journal of Dental Education, 2001, 65:249-252.

14. Cohen LA, Romberg E, Grace E. A revisitation of dental student's attitudes toward individuals with AIDS. Journal of Dental Education, 2000, 64:298-301.

15. Sears B, Ho D. HIV discrimination in health care services in Los Angeles County: the results of three testing studies. Los Angeles, Williams Institute, 2006.

16. Inada $\mathrm{K}$ et al. Factors related to the attitude of general dental practitioners toward treatment of HIV carriers. Journal of Dental Health, Tokyo, 2006, 56:240.
17. Askarian M, Mirzaei K, McLaws ML. Attitudes, beliefs, and infection control practices of Iranian dentists associated with HIV-positive patients. American Journal of Infection Control, 2006, 34:530-533.

18. Obuekwe $\mathrm{O}$ et al. Knowledge and Attitude of clinical level dental students concerning HIV/AIDS. Nigerian Dental Journal, 2007, 15:3-8.

19. Elford J et al. HIV-related discrimination reported by people living with HIV in London, UK. AIDS and Behavior, 2008, 12:255-264.

20. Stutterheim S, Bos A, Schaalma H. HIV-related stigma in the Netherlands. Maastricht, Netherlands, AIDS Fonds and University of Maastricht, 2008.

21. Hazelkorn HM. The reaction of dentists to members of groups at risk of AIDS. Journal of the American Dental Association, 1989, 119:611-619.

22. Andreoli T et al. Andreoli and Carpenter's Cecil essentials of medicine. Philadelphia, WB Saunders, 2007.

23. Fauci A et al., eds. Harrison's principles of internal medicine. New York, McGraw-Hill, 2008.

24. Little J et al. Dental management of the medically compromised patient. St Louis, Missouri, Mosby, 2007.

25. McQuistan MR et al. Dentists' comfort in treating underserved populations after participating in community-based clinical experiences as a student. Journal of Dental Education, 2008, 72:422-430. 